\title{
Is STAT3 Signaling after Focal Ischemic Stroke Beneficial
}

\author{
Johnson D, Corley C and Taylor TM* \\ Department of Biology, College of Science Engineering and Technology, Jackson State \\ University, USA
}

*Corresponding author: Tammi M Taylor, Department of Biology, College of Science Engineering and Technology, Jackson State University; Jackson, MS 39217, USA, Tel: +1-601-979-0394; Email: tammi.m.taylor@jsums.edu

\section{Mini Review \\ Volume 2 Issue 6}

Received Date: November 05, 2018

Published Date: December 28, 2018 DOI: $10.23880 /$ jenr-16000151

\section{Abstract}

Signal Transducer activator of transcription 3 (STAT3) is involved in cell signaling of neuronal stem cell proliferation, angiogenesis, and cell cycle progression. The jury is still out, about the effects of the STAT3 pathway activation after ischemia and if inhibiting or inducing this pathway leads to better functional recovery and neuronal stem cell proliferation to the penumbra. This review will better understand the role of STAT3 after focal ischemic stroke.

Keywords: Ischemia; STAT3; Penumbra

\section{Introduction}

Signal Transducer activator of Transcription 3 (STAT3) is a transcription factor and an intracellular signal transducer activated by cytokines, growth factors, and receptor- or nonreceptor-tyrosine kinases [1,2]. STATs are involved in many biological events as diverse as embryonic development, programmed cell death, organogenesis, innate immunity, adaptive immunity and cell growth regulation in organisms ranging from slime molds to insects to humans [1]. Tyrosine phosphorylation of STAT3 at Y705 is required for STAT3 activity. After phosphorylation at Y705, dimerization, and nuclear translocation, STAT3 binds to the promoters of target genes and finally induces gene expression [2].

In this review, we will discuss the importance of STAT3 and its potential neuroprotective strategies and regenerative targets that may expand to it being a molecular therapeutic target after ischemia.

\section{STAT3 after Focal Ischemia}

In developed countries, stroke is the leading cause of death and disability and beyond the immediate 4-6 h after an acute ischemic stroke, there is no known therapy that improves outcomes for this health disparity [3]. Numerous pathological events such as necrosis, apoptosis, edema, and altered cellular signaling occur after cerebral ischemia as well as after subdural hematomas [4]. Previous studies have shown that the STAT3 pathway is activated in in vitro and in vivo experimental models of stroke [5,6]. Although most studies show an increase in phosphorylation of STAT3 after stroke, there are conflicting data whether this pathway activation leads to improved neurological recovery.

Several groups have found that treating animals with hypoxic preconditioning, rhEPO, or other novel compounds increased the activation of the STAT3 pathway and improved neurological recovery after experimental stroke $[7,8]$. Furthermore, it has shown that when STAT3 phosphorylation is blocked with inhibitors AG490 or WP1066 (an analog of AG490) they found that cell death markers or functional performance were worsened [1,7-9]. The latter suggest that treatments that activate the STAT3 pathway after experimental stroke may lead to improved functional performance and decreased cell death [1]. 
On the contrary, Satriotomo, et al. (2006) found that the JAK2 inhibitor AG490 or a STAT3 siRNA after experimental cerebral ischemia decreased infarction volume, neuronal damage, apoptosis, and GFAP-positive cells $[1,10]$. These results suggest that activation of the STAT3 pathway leads to decreased cerebral recovery and that blocking this pathway leads to better neurological outcomes. Due to the controversial outcomes of STAT3 activation, further studies are required to determine the functional effects of STAT3 pathway activation following cerebral ischemia. Our group studies the effects of STAT3 on focal ischemia using neuronal STAT3 KO mice to determine if this pathway is indeed beneficial to the outcomes of regenerative repair, angiogenesis, and functional recovery.

\section{STAT3 and Neuroprotection}

Previous reports have also shown STAT3 may be involved in neuroprotection against various cerebral ischemia and brain insults [11-15]. After focal ischemia, reactive oxygen species (ROS) are produced in mitochondria. These ROS induce mitochondrialdependent apoptotic pathways [16-18]. These ROS, are removed by manganese-containing superoxide dismutase (Mn-SOD or SOD2), a primary cellular defense antioxidant enzyme specific to superoxide [16]. STAT3 regulates transcription of the Mn-SOD gene in the mouse cerebral cortex and cortical neurons [14]. Another study used Rice-Vannucci model of severe hypoxia induced insult in postnatal (P7) mice, neuronal deletion of STAT3 reduced forebrain cell death, tissue loss, microglial, and astroglial activation [19]. This data implies that STAT3 plays a critical role and may contribute to neonatal hypoxia induced-brain damage via Tyr705 phosphorylation [19]. Therefore, STAT3 is thought to play a necessary role in neuroprotection after focal ischemia.

\section{Human clinical application to STAT3}

Upon infection, inflammatory cytokines trigger cell signaling in local stem cells or differentiated cells [20]. Among other transcription factors, this eventually leads to the activation of STAT3 that mediates regenerative geneexpression programs in human disease. These genes include growth factors, cell-cycle stimulators, cell death inhibitors, and genes promoting dedifferentiation, cell motility and migration as suggested after stroke and after an immune response to infection. STAT3 has regenerative properties that has been extensively studied in hepatocyte cells in the liver [21-23] and has been proven to induce cancer [24].

\section{Adaptation to other Diseases Targeting STAT3 Molecules}

STAT3 regulates a wide spectrum of biological programs, including inflammation, tissue regeneration, cell proliferation, cell survival, cellular differentiation, angiogenesis, chemotaxis, and cell adhesion [20-24]. STAT3 plays a role in the latter processes by transcribing the expression of a variety of genes in response to specific external signals sensed by cell-surface receptors [23]. All cell types and tissues do not have the same expression patterns of these STAT3 receptors and their signaling cascade mediators. Nonetheless, STAT3 activation is highly context-dependent, which can often lead to controversial data [20]. The latter is true for the role of STAT3 in inflammation, since it can promote or suppress this process in many diseases [20-24].

\section{Conclusion}

Ischemic stroke has been shown to activate the STAT3 pathway and increase expression of STAT-3 related genes involved in cellular proliferation, differentiation, survival and inhibitory neurotransmission. However, there are conflicting reports on the effects of blocking the STAT3 pathway. Some have reported worsened neurological recovery when blocking this pathway, while others have demonstrated advantageous effects. Further investigation is needed to determine if it is feasible to selectively block only certain genes downstream of the STAT-3 pathway to prevent the deleterious effects associated with activation of this pathway while maintaining the beneficial neuroprotective effects of STAT-3 pathway activation.

Author Contributions: Conceptualization, T.T.; Methodology, T.T..; Software, T.T.; Validation, D.J.., C.C. and T.T..; Formal Analysis, T.T.; Investigation, T.T..; Resources, T.T..; Data Curation, T.T..; Writing-Original Draft Preparation, T.T.; Writing-Review \& Editing, CC, D.J, and TT; Visualization, T.T..; Supervision, T.T..; Project Administration, T.T..; Funding Acquisition, T.T

Funding: This research was funded by National Institutes of Minority Health and Health Disparities Pilot Grant PFA 398 Grant No. G12MD007581.

Acknowledgments: I would like to acknowledge Dr. Paul Tchounwou for his Research Centers for Minority Research Center pilot grant support, career development, and mentorship. 


\section{References}

1. Raible DJ, Frey LC, Brooks-Kayal AR (2014) Effects of JAK2-STAT3 signaling after cerebral insults. JAKSTAT 3: e29510.

2. Liang $\mathrm{Z}, \mathrm{Wu}$ G, Fan $\mathrm{C}, \mathrm{Xu}$ J, Jiang S, et al. (2016) The emerging role of signal transducer and activator of transcription 3 in cerebral ischemic and hemorrhagic stroke. Prog Neurobiol 137: 1-16.

3. Sun JJ, Liu Y, Ye ZR (2008) Effects of P2Y1 receptor on glial fibrillary acidic protein and glial cell line-derived neurotrophic factor production of astrocytes under ischemic condition and the related signaling pathways. Neurosci Bull 24(4): 231-243.

4. Osuka K, Watanabe Y, Usuda N, Atsuzawa K, Shima H, et al. (2013) Activation of JAK-STAT3 signaling pathway in chronic subdural hematoma outer membranes. Neurosci Lett 534: 166-170.

5. Planas AM, Soriano MA, Berruezo M, Justicia C, Estrada A, et al. (1996) Induction of Stat3, a signal transducer and transcription factor, in reactive microglia following transient focal cerebral ischaemia. Eur J Neurosci 8(12): 2612-2618.

6. Lund IV, Hu Y, Raol YH, Benham RS, Faris R, et al. (2008) BDNF selectively regulates GABAA receptor transcription by activation of the JAK/STAT pathway. Sci Signal 1(41): ra9.

7. Suzuki S, Tanaka K, Nogawa S, Dembo T, Kosakai A, et al. (2001) Phosphorylation of signal transducer and activator of transcription-3 (Stat3) after focal cerebral ischemia in rats. Exp Neurol 170(1): 63-71.

8. Chin RF, Neville BG, Peckham C, Bedford H, Wade A, et al. (2006) Incidence, cause, and short-term outcome of convulsive status epilepticus in childhood: prospective population-based study. Lancet 368(9531): 222-229.

9. Choi JS, Kim SY, Cha JH, Choi YS, Sung KW, et al. (2003) Upregulation of gp130 and STAT3 activation in the rat hippocampus following transient forebrain ischemia. Glia 41(3): 237-246.

10. Satriotomo I, Bowen KK, Vemuganti R (2006) JAK2 and STAT3 activation contributes to neuronal damage following transient focal cerebral ischemia. J Neurochem 98(5): 1353-1368.
11. Yamashita T, Sawamoto K, Suzuki S, Suzuki N, Adachi $\mathrm{K}$, et al. (2005) Blockade of interleukin-6 signaling aggravates ischemic cerebral damage in mice: possible involvement of Stat3 activation in the protection of neurons. J Neurochem 94(2): 459-468.

12. Shyu WC, Lin SZ, Chiang MF, Chen DC, Su CY, et al. (2008) Secretoneurin promotes neuroprotection and neuronal plasticity via the Jak2/Stat3 pathway in murine models of stroke. J Clin Invest 118(1): 133148.

13. Dziennis S, Jia T, Rønnekleiv OK, Hurn PD, Alkayed NJ (2007) Role of signal transducer and activator of transcription-3 in estradiol-mediated neuroprotection. J Neurosci 27(27): 7268-7274.

14. Jung JE, Kim GS, Narasimhan P, Song YS, Chan PH (2009) Regulation of Mn-superoxide dismutase activity and neuroprotection by STAT3 in mice after cerebral ischemia. J Neurosci 29(21): 7003-7014.

15. Jung JE, Kim GS, Chan PH (2011) Neuroprotection by interleukin- 6 is mediated by signal transducer and activator of transcription 3 and antioxidative signaling in ischemic stroke. Stroke 42(12): 35743579.

16. Chan PH (1996) Role of oxidants in ischemic brain damage. Stroke 27(6): 1124-1129.

17. Sugawara T, Fujimura M, Morita-Fujimura $Y$, Kawase M, Chan PH (1999) Mitochondrial release of cytochrome c corresponds to the selective vulnerability of hippocampal CA1 neurons in rats after transient global cerebral ischemia. J Neurosci 19(22): RC39.

18. Stadtman ER, Levine RL (2003) Free radical-mediated oxidation of free amino acids and amino acid residues in proteins. Amino Acids 25(3-4): 207-218.

19. Hristova M, Rocha-Ferreira E, Fontana X, Thei L, Buckle R, et al. (2016) Inhibition of Signal Transducer and Activator of Transcription 3 (STAT3) reduces neonatal hypoxic-ischaemic brain damage. J Neurochem 136(5): 981-994.

20. Sehgal PB (2008) Paradigm shifts in the cell biology of STAT signaling. Semin Cell Dev Biol 19(4): 329340. 


\section{Journal of Ecology \& Natural Resources}

21. Karin M, Clevers H (2016) Reparative inflammation takes charge of tissue regeneration. Nature 529: 307315.

22. Michalopoulos GK (2013) Principles of liver regeneration and growth homeostasis. Compr Physiol 3(1): 485-513.
23. Michalopoulos GK (2014) Advances in liver regeneration. Expert review of gastroenterology \& hepatology 8(8): 897-907.

24. Robinson MW, Harmon C, O'Farrelly C (2016) Liver immunology and its role in inflammation and homeostasis. Cell Mol Imm 13(3): 267-276. 